\title{
Mapping Membrane Topology Label Free and Corrected for Changes in the Refractive Index of the Membrane on a Nanometer Scale
}

\author{
Joachim Walter $^{1}$, Natalia Schiefermeier ${ }^{2}$, Nina Hobi ${ }^{3,4,5}$, Benjamin Grau ${ }^{6}$, Christine E. Bandtlow ${ }^{3}$, \\ Lukas A. Huber ${ }^{2,7}$, Rainer Uhl ${ }^{1}$, Sebastian Munck ${ }^{3,8}$ \\ ${ }^{1}$ BioImaging Zentrum of the Ludwig-Maximilians, Universität München Martinsried, München, Germany \\ ${ }^{2}$ Divisions of Cell Biology and for Neurobiochemistry, Biocenter - Innsbruck Medical University, Innsbruck, Austria \\ ${ }^{3}$ Biocenter - Innsbruck Medical University, Innsbruck, Austria \\ ${ }^{4}$ Department of Physiology and Medical Physics, Division of Physiology, Innsbruck Medical University, Innsbruck, Austria \\ ${ }^{5}$ Institut for General Physiology, University Ulm, Ulm, Germany \\ ${ }^{6}$ Department of Developmental Neurobiology, Instituto Cajal, C.S.I.C., Madrid, Spain \\ ${ }^{7}$ Austrian drug screening institute, ADSI, Innsbruck, Austria \\ ${ }^{8}$ VIB Center for the Biology of Disease, KU Leuven, Leuven, Belgium
}

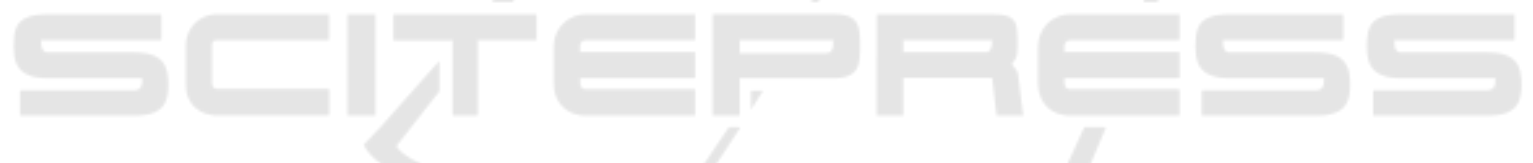

Keywords: Adhesion Sites, Membrane Dynamics, Interference Reflection Microscopy, Topography.

\begin{abstract}
The plasma membrane is the outer limit of the animal cell. As such, it is both a border separating inside from outside and a signaling platform for interactions with the surroundings. Among these interactions are extracellular matrix contacts and adhesion sites. The membrane and its contact sites together with the underlying cytoskeleton undergo constant remodeling, which leads to changes of the cell shape. In addition to spatial information micro-topographical maps provide, information about the z-dimension and describe the position of the plasma membrane with respect to the distance to a given substrate. Here we address how to measure height differences in the plasma membrane and how to create topographical maps of the plasma membrane with nanometer resolution. We address the currently used methodologies along with their advantages and drawbacks. Moreover, we delineate a label-free method to obtain topographic maps of the plasma membrane that are corrected for differences in the refractive index of the membrane utilizing an interferometric approach with multiple wavelengths and a normalization procedure to account for changes in the refractive index in the membrane.
\end{abstract}

\section{INTRODUCTION}

The cell membrane separates the intracellular content from the external environment of the cell. It forms a hull over the cytoskeleton of the cell. The shape of the cell can be compared to a tent plane spanned over its poles, which leads to bending and curvature of the membrane covering the cytoskeleton. The cytoskeleton in turn anchors the membrane to a substrate and is reported to be involved in the compartmentalization of the membrane through so called pickets and fences (Ritchie et al., 2003). The adhesion points themselves can act as signaling platforms (Cabodi et al., 2010). Cell surface proteins transduce dynamic extracellular signals to the cell interior (Spiller et al.,
2010) including interactions with the extracellular matrix, modeling multiple cellular responses including migration, differentiation and proliferation.

Per se the plasma membrane is a self-organizing organelle with strong interdependencies of its constituents which are compartmentalized through a special interaction and distribution pattern into so called nano- and microdomains (Lingwood and Simons, 2010).

In living cells, adhesion and migration are fundamental cellular processes, which undergo constant remodeling together with constant turnover of the membrane itself. Adhesion and migration are involved in all organizational levels of the organism, from single cell translocation to tissue development and movement of entire organisms. Consequently, 
adhesion and migration are fundamental aspects of development and appear early in the evolution of higher organisms (Harwood and Coates, 2004). Defects in adhesion have been implicated in several diseases, for example in neuro-developmental disorders (Olson and Walsh, 2002), and changes can be associated with the carcinogenic transformation of normal cells (Knudsen and Miranti, 2006). Considering the interdependent role of the plasma membrane, adhesion, cell surface movement, diffusion of its constituents and the interactions with the cytoskeleton have to be investigated as one complex. How cells remodel their envelope during early adhesion events and during spreading has been highlighted before (Pierres et al., 2003, Ryzhkov et al., 2010). Nanometer scale topographic maps of the membrane can be used as a read-out for this remodeling (Parthasarathy and Groves, 2004, Sugiyama et al., 2012).

A topographic map is a representation that adds the relief or height information to the classic $\mathrm{x}, \mathrm{y}$ 2-D information. Unfortunately the typical tool of the cell biologist for retrieving 3-D information, a confocal microscope does not have the resolution that is required to accurately depict the typical variations in height of cell membrane (Giebel et al., 1999). Therefore, we want to explore a technique that is not limited in depth, like for example TIRF and that ideally is label free. While there is no denying that efficient labeling techniques are available, these techniques are offset by the time and resources required to attach the probe to the biomolecule of interest. The quality of the technique is depending on the quality of the label and the labeling process. In contrast, to this previously used interferometric methods are label free. Here we present an interferometric method that uses a multi wavelength normalization procedure. By applying this new method we are the first to compensate for artifacts, which are introduced by differences in the refractive index of the membrane, e.g. at sites of adhesion between the membrane and the substrate and thus get more reliable results for creating microtopographical maps of adhering cells.

\section{DIFFERENT TECHNIQUES TO CREATE TOPOGRAPHIC MAPS}

Several procedures can be applied to investigate the membrano-cytoskeletonal complex reorganization via topographic maps on a nanometer scale. Among them are Atomic Force Microscopy (AFM), Total Internal Reflection Fluorescence Microscopy (TIRF); Surface Plasmon Resonance (SPR), Fluorescence Interference Contrast (FLIC), Point Spread Function (PSF) fitting, Foerster Resonance Energy Transfer (FRET) and micro-interference methods.

\subsection{Atomic Force Microscopy}

Atomic Force Microscopy (AFM) involves probing a surface, e.g. a cell surface by scanning a sharp tip over the cell. In AFM, the interaction between the specimen and the tip is measured by the deflection of the cantilever (the tip holder) (Zhang et al., 2010). A topographic map of the cellular membrane can be created using AFM (Duman et al., 2010). The limitations of AFM include the time-resolution as a consequence of the scanning process and the fact that only the top membrane is accessible to the scanning probe.

\subsection{Total Internal Reflection Fluorescence}

In Total Internal Reflection Fluorescence (TIRF) microscopy light is projected under an angle of total internal reflection on an interface from higher to lower refractive index. This process initiates an evanescent wave that can be used for excitation of fluorescence. The intensity of the fluorescent signal scales exponentially with the distance from the interface (Axelrod et al., 1984). This technique has been used to investigate the adhesion contacts and membrane dynamics (Reichert and Truskey, 1990, Snijder-Van As et al., 2009). The artifacts that can occur include interference fringes due to laser light being scattered at non-uniformities in the light path and anisotropic blur due to a mismatch in the refractive index between the specimen and the surrounding medium.

TIRF based topographic maps encounter the problem decreasing fluorescence intensity with increasing distance from the interface; therefore, making measurements more difficult and more inaccurate the further away the sample is from the interface. The penetration depth depends on the wavelength and the incident angle, which means that measurements in practice are limited to $\sim 200 \mathrm{~nm}$ above the coverslip (Mattheyses and Axelrod, 2006) which however was sufficient for dynamic cell measurements (Ryzhkov et al., 2010). 


\subsection{Surface Plasmon Resonance}

Surface Plasmon Resonance (SPR) measurements are another possibility for creating topographic maps and have been successfully used in the past to create topographic maps (Giebel et al., 1999). SPR are electromagnetic waves, which are traveling parallel to a metal or dielectric surface, if the frequency of the photons matches the frequency of surface electrons. SPRs are changed upon changes to the dielectric surface like absorption or binding processes. Since surface plasmons are used to establish an evanescent wave, SPR shares some similarity with TIRF microscopy.

\subsection{Fluorescence Interference Contrast}

Fluorescence Interference Contrast (FLIC) can be used to create topographic displays with nanometer resolution of the cellular envelope (Parthasarathy and Groves, 2004). Since FLIC occurs only by the interference of a light emitted by a fluorophore with its reflection in vicinity of a reflecting surface (Lambacher and Fromherz, 1996) it is not very commonly used for biological measurements.

\subsection{Point Spread Function Shape based Methods}

Another way to deduce height information on the nanometer scale has been applied for single particle tracking (Toprak et al., 2007). Here, the path of the single molecule is corrected based on information derived from the microscopic image itself and the way the light is diffracted.

For a given spot, typically the shape of the Point Spread Function (PSF) changes with height, which means for an object with a known shape and a corresponding 3-D PSF, that the z-position can be measured.

Different attempts have been made so far to use this paradigm. One way is to use a double plane detection scheme, which allows imaging of different optical planes at the same time allowing the 3-D information of the PSF to be fitted. Another method is to use a cylindrical lens as it changes the 3-D PSF due to an introduced astigmatism and allows direct conclusions to be made about the height (Kao and Verkman, 1994).

Basically, if the path of a particle is read out in 3$\mathrm{D}$, it can be corrected for movements in 3-D; however, a topographic map would not necessarily be created. This can be achieved using the 3-D detection scheme for super-resolution microscopy images as described by (Huang et al., 2008, Juette et al., 2008). The quality of the measurement depends on the quality of the PSF fit.

Concerning maps created by PSF based methods; differences in the refractive index of the membrane will as well influence the PSF. These distortions of the PSF are difficult to compensate for. Moreover, if a complete topographic map is measured on living cells with a super-resolution method, the timing can become problematic, as a multitude of images need to be acquired for these methods (Schermelleh et al., 2010). Another difficulty is that single molecule super-resolution methods are pointillistic and depend on the labeling density, which means they leave gaps, requiring interpolation of the missing data (Galbraith and Galbraith, 2011).

\subsection{Fluorescent Resonant Energy Transfer}

FRET provides information about the interaction between proteins at a resolution of a few nanometers. As such, it is used for surface interactions of proteins and was used to some extent to create topographic maps (Parthasarathy and Groves, 2004). However, due to the limited range of the resonant energy transfer, the answer provided by this technique was a contact or no contact answer and not a differentiated read-out describing the topography of the cell membrane over a longer ranges.

\subsection{Interferometric Methods}

Interferometric methods represent another potential strategy for the creation of topographic maps. Both interference reflection microscopy (IRM) and reflection interference contrast microscopy (RICM) have been applied to investigate minute changes in the height of membranes at nanometer resolution (Weber, 2003). For example, these techniques were used to investigate the dynamics of adhesion of fibroblasts to several surfaces (e.g. see (Fang et al., 2005)). However, repeatedly changing from dark to bright due to the oscillatory nature of the interference pattern over longer distances creates some ambiguity due to lack of an absolute intrinsic reference. This problem has also limited the possibility for investigation of the z-direction of objects with unknown shape. Nevertheless, recent advantages in RICM have shown that absolute distance measurements are possible in floating vesicles with the application of more wavelengths and quantitative image analysis (Schilling et al., 
2004). Consequently dual wavelength or DWreflection interference microscopy is used recently for adhesion, vesicle dynamics and further developments of the technique (for examples see (Contreras-Naranjo and Ugaz, 2013, Limozin and Sengupta, 2009, Monzel et al., 2009, Mundinger et al., 2012, Sugiyama et al., 2012)). One of the advantages of the interference methods is that they allow for label free investigation of the cell membrane. However, earlier reports have shown that interference microscopy and measurements of adhesion force were not in agreement (Zheng et al., 1994) and that the refractive index within the cell membrane can vary, especially at adhesion sites (Iwanaga et al., 2001). According to the Fresnel equations (Hecht, 2002) changes in the refractive index of the membrane lead to changes in reflectivity of the membrane. Along the same lines, it was shown that the reflectivity at adhesion sites can be higher (Atilgan and Ovryn, 2011). Moreover, we could not fit DW-RICM measurements of cellular process to the theory. In order to overcome these problems and to be able to create absolute, correct and label free topographic maps that can universally be applied to cells, DW-RICM measurements need to be corrected for differences in the refractive index.

Here we delineate a method to correct for differences in the refractive index of the membrane using a normalization procedure based on multiple wavelengths. We provide a theoretical background and show the proof of principle using a cellular process.
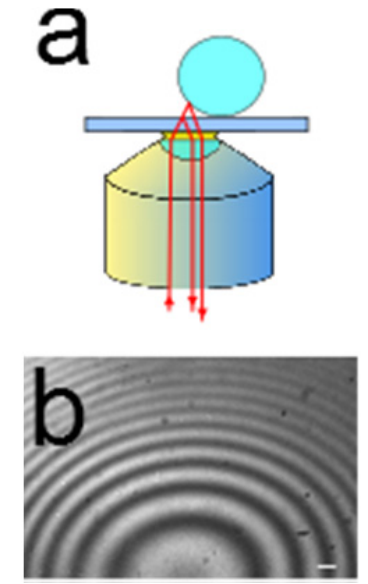

Figure 1: (a) schematic of reflective interference imaging of a glass bead. (b) interference fringes of a glass bead imaged with the setup. Scale bar $=5 \mu \mathrm{m}$.

\section{MATERIALS AND METHODS}

The images were acquired using a Zeiss Axiovert $200 \mathrm{M}$ equipped with a $63 \mathrm{x}$ oil (NA 1.4), a $100 \mathrm{x}$ oil (NA 1.45) objective lens and a RT monochromatic camera (diagnostic instruments). The setup was driven by the Metamorph software (Universal Imaging). A 50/50 beam splitter was used for reflected light illumination and to allow collection of the reflected light. A HBO lamp was used as light source using Köhler illumination and aperture and field diaphragms relatively closed to restrict the light exposure of the sample. Interference filters (center wavelength as indicated) were used in the parallel part of the illumination path to create monochromatic light. A motorized filter wheel was used to switch between the wavelengths.

The rat CNS neuroblastoma cell line B104 (Schubert et al., 1974) was grown at $8.5 \% \mathrm{CO}_{2}$ in DMEM (Invitrogen) supplemented with $10 \%$ heatinactivated fetal calf serum and $1 \%$ antibiotics (Sigma; 10,000 units penicillin $/ \mathrm{ml}$ and $10 \mathrm{mg}$ streptomycin $/ \mathrm{ml}$ ). Cultures were passaged once a week at approximately $70-80 \%$ confluence and the growth medium was changed every two days. B104 cells were plated at a density of 3,000 cells $/ \mathrm{cm}^{2}$ in growth-supplemented medium on glass bottom tissue-culture dishes (Sarstedt, Newton, NC), which coated with poly-D-lysine (Sigma) and laminin (Sigma). A heated stage and a cover were used to conserve optimal conditions for cell survival and growth (Carl Zeiss, Jena).

Beads of approximately $2 \mathrm{~mm}$ diameter with unmarked surfaces were created from glass droplets with a Bunsen burner.

Images were analyzed using ImageJ (1.44 National Institutes of Health USA, http://rsb.info.nih.gov/ij), with custom written macros. Models were calculated using Mathcad (13, Mathsoft Engineering \& Education, Cambridge MA) and Excel (Microsoft, Redmond).

For clarity, the background in the images with the growth cones has been removed based on the phase contrast image. Background in the acquired images was measured with a region of interest in the vicinity of the object. Images of Q'1, Q'2 and Q'3 were derived according to the theory section. For the creation of the height maps, values were rounded and compared to a theoretical look up table generated in Excel. Correlation plots were created using the cytofluorogram module in Jacob using Fiji (Bolte and Cordelieres, 2006, Schindelin et al., 2012). 


\section{RESULTS}

\subsection{Theory of Refractive Index-free Interferometric Measurements}

In interference microscopy, the sample is illuminated in reflective mode. Part of the light is reflected at the interface between the coverslip and the medium with a lower refractive index (interface 1). At the interface between the medium and the cell membrane, which has a higher refractive index, again part of the light is reflected (interface 2). These two reflected beams form the interference pattern that is observed as a function of the distance between the coverslip and the cell membrane. The intensity distribution of the interference pattern for a given wavelength was previously described (Schilling et al., 2004):

$$
I(h(x, y), \lambda)=I_{1}+I_{2}+2 \sqrt{I_{1} I_{2}} \cos \left(\frac{4 \pi n h(x, y)}{\lambda}+\delta\right)
$$

with $\mathrm{I}(\mathrm{h}(\mathrm{x}, \mathrm{y}),, \lambda)$ being the intensity on - the interferogram corresponding to a point whose lateral position is defined by the coordinates $(x, y)$ and the height of the membrane above the coverslip is given by $h(x, y) . \quad I_{1}$ and $I_{2}$ are the intensities of the beams reflected from interface 1 and interface 2, respectively.

$\delta$ is the difference of the phase shifts $\delta_{1 / 2}$ both beams undergo upon reflection. $\delta_{1 / 2}$ is 0 if the beam is reflected from an interface to a medium with lower refractive index, such as interface 1 . It is $\pi$ if the beam is reflected from a medium with lower refractive index such as interface 2 (Hecht, 2002). Hence, in this application

$$
\delta=\pi
$$

The intensity distribution further depends on $n$ being defined as the refractive index of the medium and $\lambda$, the wavelength of the illumination light.

A glass bead was used as a test object as it has a higher refractive index than water and a known shape, permitting analysis of the interference pattern under controlled conditions. As expected, the glass bead shows the characteristic Newton fringes (see Figure 1). When two wavelengths were applied, the resulting pattern fits nicely to the relation given in (1). When we consequently investigated cellular processes we have observed that the phase shift and the intensity do not match the relation given in (1). This finding confirms previously published data, indicating the variability of the reflection coefficient especially at focal adhesion sites (Atilgan and Ovryn, 2011, Iwanaga et al., 2001).
Consequently, our aim was to find a way to measure the height of the membrane above the coverslip without relying on the assumption of a constant reflectivity of the plasma membrane.

In Equation (2), $I_{1 / 2}$ can be written as

$$
I_{1 / 2}=a \cdot R_{1 / 2}
$$

$a$ being the intensity of the incoming light and $R_{I / 2}$ the reflectivity of the respective interface.

We used three wavelengths to correct for the inhomogeneities in the reflectivity. For wavelength $\lambda_{\alpha}$, inserting equations (2) and (3) into (1) yields

$$
I\left(h, \lambda_{\alpha}\right)=a_{\alpha} \cdot\left[R_{1}+R_{2}+2 \sqrt{R_{1} R_{2}} \cos \left(\frac{4 \pi n h}{\lambda_{\alpha}}+\pi\right)\right]
$$

Similar equations hold for wavelengths $\lambda_{\beta}$ and $\lambda_{\gamma}$.

Scaling and subtracting the intensities for different wavelengths cancels out the summand $R_{I}+R_{2}$.

$$
\begin{aligned}
& I\left(h, \lambda_{\alpha}\right)-\frac{a_{\alpha}}{a_{\beta}} I\left(h, \lambda_{\beta}\right) \\
& =2 a_{\alpha} \sqrt{R_{1} R_{2}} \cdot\left[\cos \left(\frac{4 \pi n h}{\lambda_{\alpha}}+\pi\right)-\cos \left(\frac{4 \pi n h}{\lambda_{\beta}}+\pi\right)\right] \\
& =2 a_{\alpha} \sqrt{R_{1} R_{2}} \cdot\left[\cos \left(\frac{4 \pi n h}{\lambda_{\beta}}\right)-\cos \left(\frac{4 \pi n h}{\lambda_{\alpha}}\right)\right]
\end{aligned}
$$

Finally, division by a similar expression with a third wavelength eliminates the term $\sqrt{R_{1} R_{2}}$.

$$
\begin{gathered}
Q_{\alpha \beta \gamma}=\frac{I\left(\left(h, \lambda_{\alpha}\right)-\frac{a_{\alpha}}{a_{\beta}} I\left(h, \lambda_{\beta}\right)\right.}{I\left(h, \lambda_{\alpha}\right)-\frac{a_{\alpha}}{a_{\gamma}} I\left(h, \lambda_{\gamma}\right)} \\
=\frac{\cos \left(\frac{4 \pi n h}{\lambda_{\beta}}\right)-\cos \left(\frac{4 \pi n h}{\lambda_{\alpha}}\right)}{\cos \left(\frac{4 \pi n h}{\lambda_{\gamma}}\right)-\cos \left(\frac{4 \pi n h}{\lambda_{\alpha}}\right)}
\end{gathered}
$$

As the denominator reaches zero, $Q_{\alpha \beta \gamma}$ contains reoccurring discontinuities which can be avoided by defining the denominator as

$$
D=\left[\begin{array}{l}
\left.\left[I\left(h, \lambda_{\alpha}\right)-\frac{a_{\alpha}}{a_{\beta}} I\left(h, \lambda_{\beta}\right)\right]^{2}+\left[I\left(h, \lambda_{\alpha}\right)-\frac{a_{\alpha}}{a_{\gamma}} I\left(h, \lambda_{\gamma}\right)\right]^{2}\right]^{\frac{1}{2}} \\
+\left[\frac{a_{\alpha}}{a_{\beta}} I\left(h, \lambda_{\beta}\right)-\frac{a_{\alpha}}{a_{\gamma}} I\left(h, \lambda_{\gamma}\right)\right]^{2}
\end{array}\right.
$$

and defining new quotients as 


$$
\begin{gathered}
Q_{1}^{\prime}=\frac{I\left(\left(h(x, y), \lambda_{\alpha}\right)-\frac{a_{\alpha}^{2}}{a_{\beta}^{2}} I\left(h(x, y), \lambda_{\beta}\right)\right.}{D} \\
Q_{2}^{\prime}=\frac{I\left(\left(h(x, y), \lambda_{\alpha}\right)-\frac{a_{\alpha}^{2}}{a_{\gamma}^{2}} I\left(h(x, y), \lambda_{\gamma}\right)\right.}{D} \\
Q_{3}^{\prime}=\frac{\frac{a_{\alpha}^{2}}{a^{2}} I\left(h, \lambda_{\beta}\right)-\frac{a_{\alpha}^{2}}{a_{\gamma}^{2}} I\left(h, \lambda_{\gamma}\right)}{D}
\end{gathered}
$$

The expressions $\frac{a_{\alpha}}{a_{\beta}}$ and $\frac{a_{\alpha}}{a_{\gamma}}$ can be measured in areas of the images, where only the interface between the coverslip to the medium contributes to the signal (cell-free areas) as from equation (3) it follows

$$
I_{1 \alpha} / I_{1 \beta}=a_{\alpha} \cdot R_{1} / a_{\beta} \cdot R_{1}=\frac{a_{\alpha}}{a_{\beta}}
$$

An instructive display of the different wavelengths $(540 \mathrm{~nm}, 600 \mathrm{~nm}$ and $685 \mathrm{~nm})$ illuminating the bead (Figure 2 a-d), their relation to the height of the bead and the relative intensity of the consequent quotients Q'1, Q'2 and Q'3 in dependence of the measured height of the glass bead can be seen in Figure 2 e-h. The height of the lower hemisphere of the glass bead can be calculated and is a function of the radius $r$ and distance $\mathrm{x}$ from the center where the glass bead touches the coverslip (see (12)).

$$
r-\sqrt{r^{2}-x^{2}}=h
$$

Overall, the intensity distributions follow the theoretical descriptions. The different wavelengths in Figure 2d show the described phase shift (Schilling et al., 2004). For the RICM, the intensity changes with height of the bead, which is in agreement with previous findings (ContrerasNaranjo and Ugaz, 2013). In contrast to DW-RICM our approach normalizes for this effect.

\subsection{Practical Test of Refractive Index Free Interferomtric Measurements}

Next the growth cone of a B104 cell, a widely used neuronal model (Schubert et al., 1974), was investigated as a more physiologically relevant model (Figure 3). The sample was imaged with the same three wavelengths as the glass bead described above (Figure 3 a-c). The equations above were applied to calculate Q'1, Q'2 and Q'3 (Figure 3 d-f).
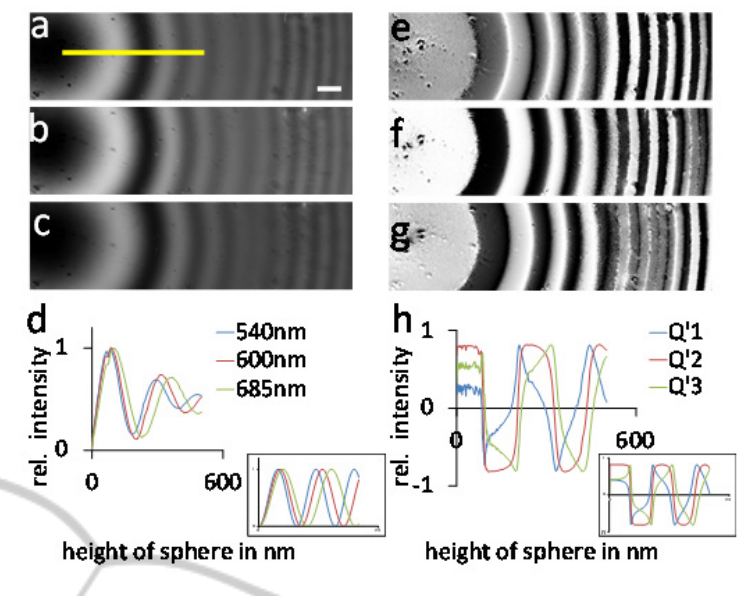

Figure 2: Testing multi-wavelength interference microscopy on glass beads. (a-c) interferometric images of the glass bead using light of $540 \mathrm{~nm} ; 600 \mathrm{~nm}$ and $685 \mathrm{~nm}$ wavelength. (d) is an intensity profile of the pictures (a), (b) and (c) from the region indicated by the yellow line in (a). The $\mathrm{x}$ axis represents the height if the glass bead based on equation 12. The length of the region is about $30 \mu \mathrm{m}$. Insert shows theoretical values (e-g) depicts the calculated quotients Q'1-Q'3 based on (a-c). (h) the relative intensity distribution of $\mathrm{Q}^{\prime}{ }_{1}, \mathrm{Q}^{\prime}{ }_{2}$ and $\mathrm{Q}^{\prime}{ }_{3}$ of (e-f) similar to (d). Insert shows theoretical values. Scale bar $=5 \mu \mathrm{m}$.

To test DW-RIM under these conditions, a rectangle of 50x50 pixels in the growth cone was selected and is displayed in a correlative graph. It can be seen that the points do not strongly follow a pattern and the characteristic curl shaped relation of (Schilling et al., 2004) does not become visible. Using the same approach with our method and using the Q'1 and Q'2 (Figure $3 \mathrm{~h}$ ), ) the expected oval shaped relation of the two Q's becomes recognizable. Next, we explored calculating a 3-D topography of the membrane. As the concatenation of $Q_{1}^{\prime} \circ Q_{3}^{\prime}$ can be used for calculating the height in the range from 0 to $240 \mathrm{~nm}$, which proved to be sufficient in this case, a look-up table was generated and applied to the values measured from the growth cone. Therefore, an image with grey values representing the height in $\mathrm{nm}$ can be deduced and is displayed as a surface plot next to the phase contrast image of the growth cone (Figure 3i,j). The result is in agreement with earlier publications (Giebel et al., 1999). It was not possible to map all pixels in the look-up table with the measured values, which is probably due to noise in the system; these values were consequently set to zero. The overall noise was relatively low as can be seen by the minimal variation in the relation of 
Figure 3h, which depicts depicting the oval shaped relation of Q'1 and Q'2 with little variation. To demonstrate the dynamic behavior of the membrane and the height changes, the same growth cone was imaged s20s later (Figure 3 i,j). Figure 3 k,l shows another example of a growth cone demonstrating that the height distributions can vary in different neuronal processes.

Taken together, we present a multi-wavelength extension of RICM: MIRM. By applying different wavelengths for illumination, we can reconstruct the refractive index corrected 3-D shape of the cellular membrane.

\section{CONCLUSIONS}

Here we have described different ways to measure membrane topography presenting the advantages and limitations of several methodologies. We highlight the benefits of label free interferometric measurements, which can measure over a longer range then TIRF, SPR, FLIC and FRET measurements. Different then AFM interferometric methods can probe the cell membrane, where the adhesion sites are located. For imaging without gaps the PSF based techniques require dense labeling, but need to be read out in a non-overlapping way. This requires a single molecule approach, which entails the acquisition of much more images as compared to our method. Another advantage of interferometric methods is that they can be designed to be compatible with labeling techniques and thus allow for a higher degree of multiplexing. So far the labeling independent interferometric methods have suffered from ambiguity due to changes in the refractive index. Here we show that when corrected for differences in refractive index, unambiguous imaging of membrane topologies can be achieved on a widefield system. In comparison to the earlier described method of (Schilling et al., 2004), the method we propose has the advantage that differences in the refractive index of the membrane can be cancelled out. This effect can be seen in the comparison of Figure $3 \mathrm{~g}$ and $\mathrm{h}$ and in the fact that a clear relationship only becomes visible when differences in the refractive index are cancelled out, emphasizing that the differences in the refractive index are not only drastic but can also be corrected. Furthermore, the framework presented here can easily be expanded to longer distances by applying more wavelengths and using different wavelength triplets for correction.

We have noticed that the method is sensitive to
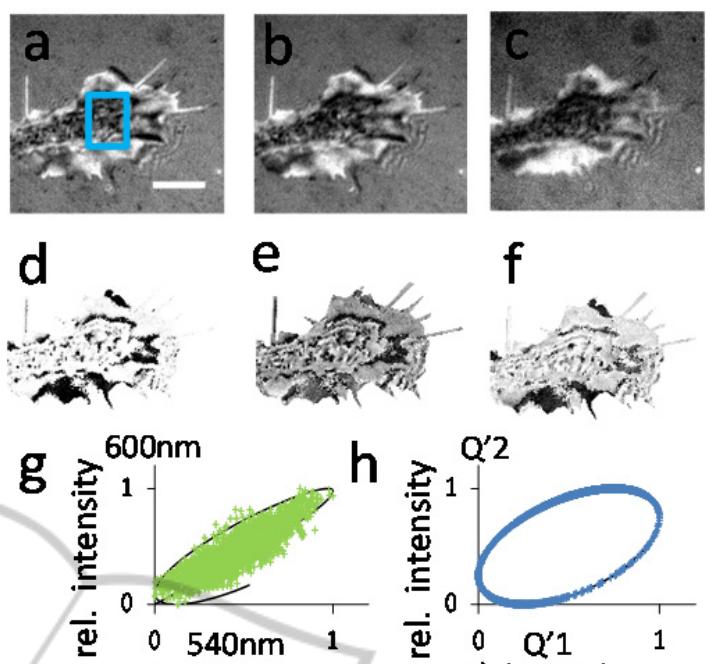

rel. intensity
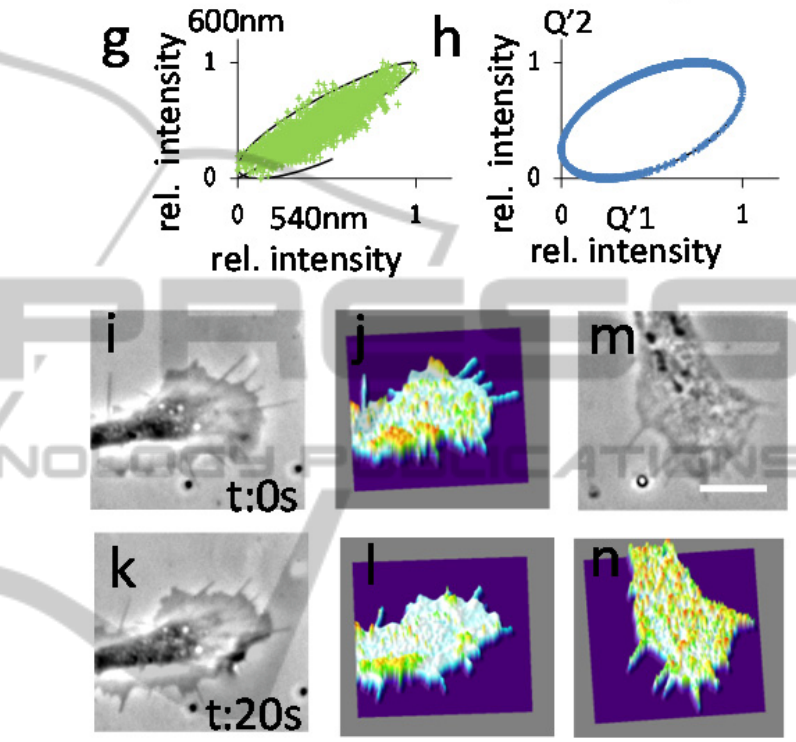

Figure 3: Testing multi-wavelength interference microscopy for mapping height profiles in a refractive index free manner on cellular processes. (a-c) interferometric images of a neuronal growth cone using light of $540 \mathrm{~nm} ; 600 \mathrm{~nm}$ and $685 \mathrm{~nm}$ wavelength. (d-f) depicts the calculated quotients Q'1-Q'3 based on (a-c). (g) correlation of the normalized intensities of (a) and (b) of the region indicated by the blue rectangle in (a). Theory in black. (h) correlation of Q'1 and Q'2 like in (g). Theory in black. (i) phase contrast picture of a neuronal process. (j) is a reconstruction of the membrane topology of the cellular process in (i). (k) the same growth cone as in (i) 20 seconds later. (1) 3-D topographical map of the growth cone in $(\mathrm{k}) .(\mathrm{m})$ another example of a neuronal growth cone in phase contrast. (n) 3-D topographical map of the growth cone in $(\mathrm{m})$.

For the reconstruction the relation between $\mathrm{Q}_{1}{ }_{1}$ and $\mathrm{Q}_{3}$ with the wavelength triplet $540 / 600 / 685 \mathrm{~nm}$ has been used.

Scale bars $=5 \mu \mathrm{m}$ color/height range in $(\mathrm{j}, 1$ and $\mathrm{n})$ from purple $=$ zero to peaks in red $=200 \mathrm{~nm}$.

background disturbances. Therefore, subtraction and the relative normalization of the individual wavelengths are critical for correct calculation.

Here the corrected membrane topography has been reconstructed with an unprecedented $\sim 30 \mathrm{~nm}$ 
resolution (Fig. 3). Theoretically, a much better resolution in z-direction is possible than shown in Figure 3. The limited resolution here is mainly due to the use of the look up table. That relative differences of $\sim 5 \mathrm{~nm}$ can be practically be achieved using interference measurements has been demonstrated earlier (Llobet et al., 2003). The achievable practical resolution depends on the sensitivity of the detector and the wavelengths used. Due to the nonlinearity in the obtained relation after correction for the refractive index, the achievable resolution cannot be equal at all distances. Like in the PSF based methods, our implementation leaves gaps; however these could be improved when the measurements are fitted to the quotients instead of using a look-up table.

It should to be emphasized that correction for differences in height is needed for single particle tracking. Simple arithmetic indicates that the error calculated for a movement in the membrane is about $12 \%$, e.g if a height change of $150 \mathrm{~nm}$ is ignored over a $300 \mathrm{~nm}$ distance. We believe an independent measurement, undisturbed from refractive index changes, is achievable with a label free technique and is a good alternative to the currently used methods for the correction (Kao and Verkman, 1994, Toprak et al., 2007).

One of the advantages of the described method is its speed. The three wavelengths for reflective imaging can easily be acquired in the millisecond range. The analysis can be implemented for almost instantaneous results (see as well (Contreras-Naranjo and Ugaz, 2013)). The fact that the method is label free means that it is relatively cheap and is making it attractive for large scale quantitative analysis and screening tasks. One potential application we see is screening for the transition between mesenchymal and amoeboid modes of migration of tumor cell upon drug treatment. This transition is relevant for potential treatment as it has been put in the context of tumor invasiveness and aggressiveness (Kosla et al., 2013, Pankova et al., 2010). Another application field is neuroscience as exemplified here. Neuronal growth cones are the growing tips of neurons. These exhibit fast remodeling of the cytoskeleton and the membrane when growing towards their targets. The dynamic of this remodeling is demonstrated by Figure $3 \mathrm{i}-1$. Moreover, that fact that there are different states of height maps is emphasized when comparing Figure 3i-l with Figure $3 \mathrm{~m}$ and $\mathrm{n}$.

Overall, we have only started to understand how cells move and migrate; therefore it is important to look at all processes and not only at the points of contact. With topographical maps, the overall remodeling of the cellular envelope with respect to their movement and interaction with the substrate can be observed. This aspect of the remodeling of membrane has been neglected so far, potentially due to the lack of good methods. However we believe it poses an important aspect of the cell and the migration process. Considering migration and focusing on the adhesion sites would be like looking solely at the feet when trying to figure out how a human walks.

The analysis of the topography of the cell membrane provides information on membrane trafficking and the remodeling of the underlying cytoskeleton as one complex that is remodeled during cellular movement. We believe that the tool presented here is a useful extension of the standard repertoire to investigate adhesion and migration and will contribute toward the illumination of these processes in the future.

\section{REFERENCES}

Atilgan, E. \& Ovryn, B. 2011. Reflectivity and topography of cells grown on glass-coverslips measured with phase-shifted laser feedback interference microscopy. Biomed Opt Express, 2, 2417-37.

Axelrod, D., Burghardt, T. P. \& Thompson, N. L. 1984. Total internal reflection fluorescence. Annu Rev Biophys Bioeng, 13, 247-68.

Bolte, S. \& Cordelieres, F. P. 2006. A guided tour into subcellular colocalization analysis in light microscopy. J Microsc, 224, 213-32.

Cabodi, S., Del Pilar Camacho-Leal, M., Di Stefano, P. \& Defilippi, P. 2010. Integrin signalling adaptors: not only figurants in the cancer story. Nat Rev Cancer, 10, 858-70.

Contreras-Naranjo, J. C. \& Ugaz, V. M. 2013. A nanometre-scale resolution interference-based probe of interfacial phenomena between microscopic objects and surfaces. Nat Commun, 4, 1919.

Duman, M., Pfleger, M., Zhu, R., Rankl, C., Chtcheglova, L. A., Neundlinger, I., Bozna, B. L., Mayer, B., Salio, M., Shepherd, D., Polzella, P., Moertelmaier, M., Kada, G., Ebner, A., Dieudonne, M., Schutz, G. J., Cerundolo, V., Kienberger, F. \& Hinterdorfer, P. 2010. Improved localization of cellular membrane receptors using combined fluorescence microscopy and simultaneous topography and recognition imaging. Nanotechnology, 21, 115504.

Fang, N., Zhu, A., Chan-Park, M. B. \& Chan, V. 2005. Adhesion contact dynamics of fibroblasts on biomacromolecular surfaces. Macromol Biosci, 5, 1022-1031.

Galbraith, C. G. \& Galbraith, J. A. 2011. Super-resolution microscopy at a glance. J Cell Sci, 124, 1607-11. 
Giebel, K., Bechinger, C., Herminghaus, S., Riedel, M., Leiderer, P., Weiland, U. \& Bastmeyer, M. 1999. Imaging of cell/substrate contacts of living cells with surface plasmon resonance microscopy. Biophys $J$, 76, 509-16.

Harwood, A. \& Coates, J. C. 2004. A prehistory of cell adhesion. Current Opinion in Cell Biology, 16, 470476.

Hecht, E. 2002. Optics, Reading, Mass., Addison-Wesley.

Huang, B., Wang, W., Bates, M. \& Zhuang, X. 2008. Three-dimensional super-resolution imaging by stochastic optical reconstruction microscopy. Science, 319, 810-3.

Iwanaga, Y., Braun, D. \& Fromherz, P. 2001. No correlation of focal contacts and close adhesion by comparing GFP-vinculin and fluorescence interference of Dil. European Biophysics Journal: EBJ, 30, 17-26.

Juette, M. F., Gould, T. J., Lessard, M. D., Mlodzianoski, M. J., Nagpure, B. S., Bennett, B. T., Hess, S. T. \& Bewersdorf, J. 2008. Three-dimensional sub-100 nm resolution fluorescence microscopy of thick samples. Nat Methods, 5, 527-9.

Kao, H. P. \& Verkman, A. S. 1994. Tracking of single fluorescent particles in three dimensions: use of cylindrical optics to encode particle position. Biophys $J, 67,1291-300$.

Knudsen, B. S. \& Miranti, C. K. 2006. The impact of cell adhesion changes on proliferation and survival during prostate cancer development and progression. J Cell Biochem, 99, 345-61.

Kosla, J., Pankova, D., Plachy, J., Tolde, O., Bicanova, K., Dvorak, M., Rosel, D. \& Brabek, J. 2013. Metastasis of aggressive amoeboid sarcoma cells is dependent on Rho/ROCK/MLC signaling. Cell Commun Signal, 11, 51.

Lambacher, A. \& Fromherz, P. 1996. Fluorescence interference-contrast microscopy on oxidized silicon using a monomolecular dye layer Applied Physics A: Materials Science \& Processing, 63, 207-216.

Limozin, L. \& Sengupta, K. 2009. Quantitative reflection interference contrast microscopy (RICM) in soft matter and cell adhesion. Chemphyschem, 10, 2752-68.

Lingwood, D. \& Simons, K. 2010. Lipid rafts as a membrane-organizing principle. Science, 327, 46-50.

Llobet, A., Beaumont, V. \& Lagnado, L. 2003. Real-Time Measurement of Exocytosis and Endocytosis Using Interference of Light. Neuron, 40, 1075-1086.

Mattheyses, A. L. \& Axelrod, D. 2006. Direct measurement of the evanescent field profile produced by objective-based total internal reflection fluorescence. J Biomed Opt, 11, 014006.

Monzel, C., Fenz, S. F., Merkel, R. \& Sengupta, K. 2009. Probing biomembrane dynamics by dual-wavelength reflection interference contrast microscopy. Chemphyschem, 10, 2828-38.

Mundinger, T. A., Sommerfeld, A., Reinehr, R., Spatz, J. P., Haussinger, D. \& Boehm, H. 2012. Investigating cell-ECM contact changes in response to hypoosmotic stimulation of hepatocytes in vivo with DW-RICM. PLoS One, 7, e48100.
Olson, E. C. \& Walsh, C. A. 2002. Smooth, rough and upside-down neocortical development. Current Opinion In Genetics \& Development, 12, 320-327.

Pankova, K., Rosel, D., Novotny, M. \& Brabek, J. 2010. The molecular mechanisms of transition between mesenchymal and amoeboid invasiveness in tumor cells. Cell Mol Life Sci, 67, 63-71.

Parthasarathy, R. \& Groves, J. T. 2004. Optical techniques for imaging membrane topography. Cell Biochemistry And Biophysics, 41, 391-414.

Pierres, A., Eymeric, P., Baloche, E., Touchard, D., Benoliel, A.-M. \& Bongrand, P. 2003. Cell membrane alignment along adhesive surfaces: contribution of active and passive cell processes. Biophysical Journal, 84, 2058-2070.

Reichert, W. M. \& Truskey, G. A. 1990. Total internal reflection fluorescence (TIRF) microscopy. I. Modelling cell contact region fluorescence. $J$ Cell Sci, 96 ( Pt 2), 219-30.

Ritchie, K., Iino, R., Fujiwara, T., Murase, K. \& Kusumi, A. 2003. The fence and picket structure of the plasma membrane of live cells as revealed by single molecule techniques (Review). Mol Membr Biol, 20, 13-8.

Ryzhkov, P., Prass, M., Gummich, M., Jac-Simon K"Uhn, J.-S., C., O. \& D"Obereiner, H.-G. 2010. Adhesion patterns in early cell spreading. J. Phys.: Condens. Matter, 194106 (8pp).

Schermelleh, L., Heintzmann, R. \& Leonhardt, H. 2010. A guide to super-resolution fluorescence microscopy. $J$ Cell Biol, 190, 165-75.

Schilling, J., Sengupta, K., Goennenwein, S., Bausch, A. R. \& Sackmann, E. 2004. Absolute interfacial distance measurements by dual-wavelength reflection interference contrast microscopy. Physical Review. E, Statistical, Nonlinear, And Soft Matter Physics, 69, 021901.

Schindelin, J., Arganda-Carreras, I., Frise, E., Kaynig, V., Longair, M., Pietzsch, T., Preibisch, S., Rueden, C., Saalfeld, S., Schmid, B., Tinevez, J. Y., White, D. J., Hartenstein, V., Eliceiri, K., Tomancak, P. \& Cardona, A. 2012. Fiji: an open-source platform for biologicalimage analysis. Nat Methods, 9, 676-82.

Schubert, D., Heinemann, S., Carlisle, W., Tarikas, H., Kimes, B., Patrick, J., Steinbach, J. H., Culp, W. \& Brandt, B. L. 1974. Clonal cell lines from the rat central nervous system. Nature, 249, 224-227.

Snijder-Van As, M. I., Rieger, B., Joosten, B., Subramaniam, V., Figdor, C. G. \& Kanger, J. S. 2009. A hybrid total internal reflection fluorescence and optical tweezers microscope to study cell adhesion and membrane protein dynamics of single living cells. $J$ Microsc, 233, 84-92.

Spiller, D. G., Wood, C. D., Rand, D. A. \& White, M. R. 2010. Measurement of single-cell dynamics. Nature, 465, 736-45.

Sugiyama, N., Asai, Y., Yamauchi, T., Kataoka, T., Ikeda, T., Iwai, H., Sakurai, T. \& Mizuguchi, Y. 2012. Labelfree characterization of living human induced pluripotent stem cells by subcellular topographic imaging technique using full-field quantitative phase 
microscopy coupled with interference reflection microscopy. Biomed Opt Express, 3, 2175-83.

Toprak, E., Balci, H., Blehm, B. H. \& Selvin, P. R. 2007. Three-dimensional particle tracking via bifocal imaging. Nano Lett, 7, 2043-5.

Weber, I. 2003. Reflection interference contrast microscopy In: PARKER, G. M. A. I. (ed.) Methods in Enzymology. ed.: Academic Press.

Zhang, Y., Hu, X., Sun, J., Shen, Y., Hu, J., Xu, X. \& Shao, Z. 2010. High-resolution imaging and nanomanipulation of biological structures on surface. Microsc Res Tech.

Zheng, J., Buxbaum, R. E. \& Heidemann, S. R. 1994. Measurements of growth cone adhesion to culture surfaces by micromanipulation. The Journal of Cell Biology, 127, 2049-2060.

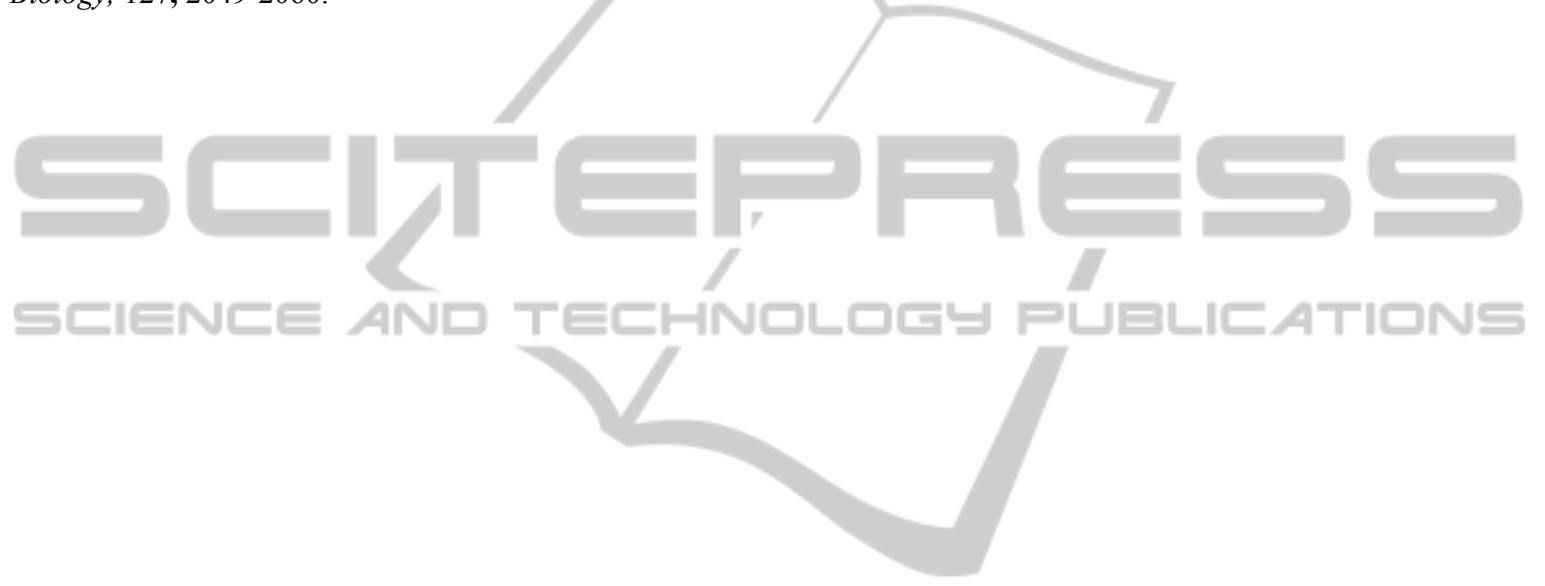

